\title{
Nonlinear Control of Chaotic Forced Duffing and Van der Pol Oscillators
}

\author{
Mohammed Alghassab1,2, Amr Mahmoud1, Mohamed A. Zohdy1 \\ ${ }^{1}$ Electrical and Computer Engineering Department, Oakland University, Rochester, MI, USA \\ ${ }^{2}$ College of Engineering, Shaqra University, Riyadh, Saudi Arabia \\ Email: maalghassab@oakland.edu,amahmoud@oakland.edu, zohdyma@oakland.edu
}

How to cite this paper: Alghassab, M., Mahmoud, A. and Zohdy, M.A. (2017) Nonlinear Control of Chaotic Forced Duffing and Van der Pol Oscillators. International Journal of Modern Nonlinear Theory and Application, 6, 26-37. https://doi.org/10.4236/ijmnta.2017.61003

Received: December 24, 2016 Accepted: February 13, 2017

Published: February 16, 2017

Copyright $\odot 2017$ by authors and Scientific Research Publishing Inc. This work is licensed under the Creative Commons Attribution International License (CC BY 4.0).

http://creativecommons.org/licenses/by/4.0/

\begin{abstract}
This paper discusses a novel technique and implementation to perform nonlinear control for two different forced model state oscillators and actuators. The paper starts by discussing the Duffing oscillator which features a second order non-linear differential equation describing complex motion whereas the second model is the Van der Pol oscillator with non-linear damping. A first order actuator is added to both models to expand on the chaotic behavior of the oscillators. In order to control the system without comprising linearization, Lyapunov non-linear control was used. A control Lyapunov function was tailored to the system. This led to improved maneuverability of the controller and the performance of the overall system. The controller was found to be highly efficient in system tracking and had swift response time. Simulations were performed on both the uncontrolled and controlled cases. Both simulation results ultimately confirmed the effectiveness of the proposed controller.
\end{abstract}

\section{Keywords}

Duffing Oscillator, Lyapunov Function, Nonlinear Control, Van der Pol Oscillator

\section{Introduction}

Duffing and Van der Pol Oscillators are typical examples of nonlinear dynamic systems and thus we will use them as a reference to test the implemented controllers. Both oscillators are good examples of periodically forced oscillators with non-linear elasticity. A Duffing oscillator can be represented by the mathematical model shown in Equation (1); on the other hand a Van der Pol Oscillator mathematical model is shown in Equation (2).

$$
\ddot{x}+\delta \dot{x}+\alpha x+\beta x^{3}=0
$$




$$
\ddot{x}+\alpha\left(x^{2}-1\right) \dot{x}+x=0
$$

A forced or driven oscillator means that a driving function of $\cos w t$ is added to Equations (1) and (2). The updated mathematical model will become as shown in Equations (3) and (4) representing the Duffing and the Van der Pol Oscillators respectively.

$$
\begin{aligned}
& \ddot{x}+\delta \dot{x}+\alpha x+\beta x^{3}=P \cos w t \\
& \ddot{x}+\alpha\left(x^{2}-1\right) \dot{x}+x=P \cos w t
\end{aligned}
$$

In our application, we apply an actuator for the oscillators and this yields the updated mathematical model that will be used in our calculations. Equation (5) represents a driven Duffing oscillator with an actuator. Equation (7) on the other hand, represents a driven Van der Pol Oscillator again with an actuator. Mathematical models of the forced Duffing and Van der Pol systems are shown respectively.

$$
\ddot{x}+\delta \dot{x}+\alpha x+\beta x^{3}=P \cos w t+u
$$

From Equation (5) the state space was deduced to be as shown below:

$$
\begin{aligned}
& \left(\begin{array}{l}
\dot{x}_{1} \\
\dot{x}_{2}
\end{array}\right)=\left(\begin{array}{c}
x_{2} \\
-\beta x_{1}^{3}-\delta x_{2}-\alpha x_{1}+P \cos w t+u
\end{array}\right) \\
& \ddot{x}+\alpha\left(x^{2}-1\right) \dot{x}+x=P \cos w t+u
\end{aligned}
$$

From Equation (7) the state space was deduced to be as shown below:

$$
\left(\begin{array}{c}
\dot{x}_{1} \\
\dot{x}_{2}
\end{array}\right)=\left(\begin{array}{c}
x_{2} \\
-\alpha\left(x_{1}^{2}-1\right) x_{2}-x_{1}+P \cos w t+u
\end{array}\right)
$$

where $\alpha, \beta, \delta$ and, $w$ are all given constants [1]. $u$ is the given actuator. Equation (9) represents the mathematical model of the actuator.

$$
\dot{u}+\beta u=g
$$

In a previous work [2], we presented a technique to control a chaotic Duffing Oscillators using recursive back stepping Control [3]. In this paper, we will continue on the work that was presented earlier by showing a solution for the Van der Pol oscillator as well. The work presented in this paper will also deal with the mathematical model when an actuator is added as shown in Equations (6) and (8). The next section will list and describe some of the related works that have been done in this field. The following section will describe our proposed method with some of the generated results. The paper is then ended with a conclusion and a future work.

\section{Related Work}

There are numerous applications for Duffing and Van der Pol Oscillators. For example, the Duffing oscillator has been used to do chirp signal detection [4]. It is also widely used in the signal communication domain such as in the secure communication field as shown in [5] and weak signal detection as shown in [6] 
and [7]. It has also found its ways to marine applications such as ship propeller blade number recognition as shown by Wang et al. in [8]. Van der Pol oscillator has had less application examples than the Duffing oscillator nevertheless it still found its way into numerous domains such as in the medical field where was used in the modelling of the cardiac pulse as shown in [9]. Another example is the use of the Van der Pol oscillator in the modeling of the dust density wave fields as shown in [10].

There has been some work done on the control of a Duffing Oscillator. Kuo et al. [11] used a fuzzy sliding controller. The control rules were based on the Lyapunov stability theorem, simulation results proved that it can successfully control the system even with the presence of chaos. Alexander Jimenez-Triana et al. [12] also presented chaos control for a Duffing system but this time using impulsive parametric perturbations. The presented approach has been established based on Melnikov's method [13] and the authors confirmed the success of the proposed method using numerical simulations.

The authors in [14] investigated the dynamic characteristics of the Van der Pol system with added delay. The authors found that Hopf bifurcation occurs from trivial equilibrium when the delay passes through critical values. The authors then found the critical values and their relationship with the system parameters. The authors proved their results using numerical results. Yang [15] et al. studied chaos control in a Van der Pol system with nonlinear force and two forcing excitations. The authors proved their results using numeral simulation. The authors concluded that chaotic motions are controllable by adjusting the phase difference and the amplitude of the second excitation force. Lastly, Van der Pol system control was also done using bifurcation such as the work that was done by M. Xiao et al. [16].

\section{Proposed Method and Chaos Analysis}

\subsection{Duffing Analysis}

Equation (5) represents the forced Duffing oscillator alongside an actuator. Let the error $e$ be defined as the $x_{\text {desired }}-x$. This means that $\ddot{e}=\ddot{x}_{\text {desired }}-\ddot{x}$.

Let $r$ be defined as shown in Equation (10) thus $\dot{r}$ can be defined as shown in Equation (11) and finally $v$ and $\dot{v}$ are defined as shown in Equations (12) and (13) respectively. $\gamma_{1}$ and $\gamma_{2}$ are constants representing tunable parameters.

$$
\begin{aligned}
r & =\gamma_{1} e+\gamma_{2} \dot{e} \\
\dot{r} & =\gamma_{1} \dot{e}+\gamma_{2} \ddot{e} \\
v & =\frac{1}{2} r^{2} \\
\dot{v} & =r \dot{r}
\end{aligned}
$$

Substituting $r$ and $\dot{r}$ in Equation (13) will yield $\dot{v}=\left(\gamma_{1} e+\gamma \dot{e}\right)\left(\gamma_{1} \dot{e}+\gamma_{2} \ddot{e}\right)=-k v \quad$ (Lyapunov variable). 
This means that $\dot{v}=\frac{-k}{2}\left(\gamma_{1} e+\gamma_{2} \dot{e}\right)$.

Rearranging Equation (5) will yield the following: $\ddot{x}=-\delta \dot{x}-\alpha x-\beta x^{3}+P \cos w t+u$, substituting the variables and rearranging will yield to Equation (14) as shown below.

$$
u=\frac{k}{2} \frac{\gamma_{1}}{\gamma_{2}} e+\frac{k}{2} \dot{e}+\frac{\gamma_{1}}{\gamma_{2}} \dot{e}+\ddot{x}_{\text {desired }}+\delta \dot{x}+\alpha x+\beta x^{3}-P \cos w t
$$

Now if we suppose that $v=\frac{1}{2} r^{4}$ and we follow the same procedure, $u$ will be generated as shown in Equation (15).

$$
u=\frac{k}{4} \frac{\gamma_{1}}{\gamma_{2}} e+\frac{k}{4} \dot{e}+\frac{\gamma_{1}}{\gamma_{2}} \dot{e}+\ddot{x}_{\text {desired }}+\delta \dot{x}+\alpha x+\beta x^{3}-P \cos w t
$$

The system performance was captured at the values below as shown in Table 1 . The following figures show the results of the presented control system. Figure 1 shows a Duffing Actuator Position on Velocity with a stable trajectory for Duffing equation with sinusoidal drive using the Grapher application. On the other hand, Figure 2 shows the Duffing Actuator Position on Velocity Unstable Chaotic trajectory for Duffing equation with sinusoidal drive also using the Grapher application. In both cases, it is assumed that $x$ is $\theta$ and $y$ is $v$. Figure 3 shows a Duffing Stable solution at 25 in poles with comparison to a reference sinusoidal drive. Figure 4 on the other hand shows a Duffing uncontrollable system when compared to a reference sinusoidal drive. Looking at the position trajectory in Figure 5, it can be noticed that it is uncontrollable position with a sinusoidal drive using the Grapher application, it is also assumed here that that $x$ is $\theta$ and $y$ is $v$. Finally Figure 6 , shows an unstable velocity in comparison to the reference sinusoidal drive.

Table 1. System Performance captured at these specific values.

\begin{tabular}{cccccc}
\hline $\boldsymbol{\alpha}$ & $\boldsymbol{\delta}$ & $\boldsymbol{\beta}$ & $\gamma_{1}$ & $\gamma_{2}$ & $\boldsymbol{k}$ \\
\hline 0.0003 & 0.03 & 0.0002 & 1 & 0.7 & 190 \\
\hline
\end{tabular}

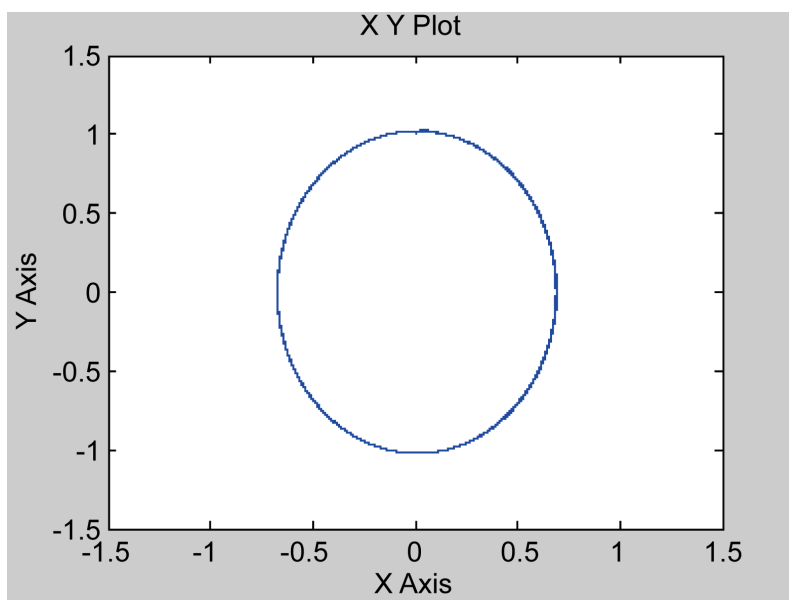

Figure 1. Duffing Actuator Position on Velocity Stable. 


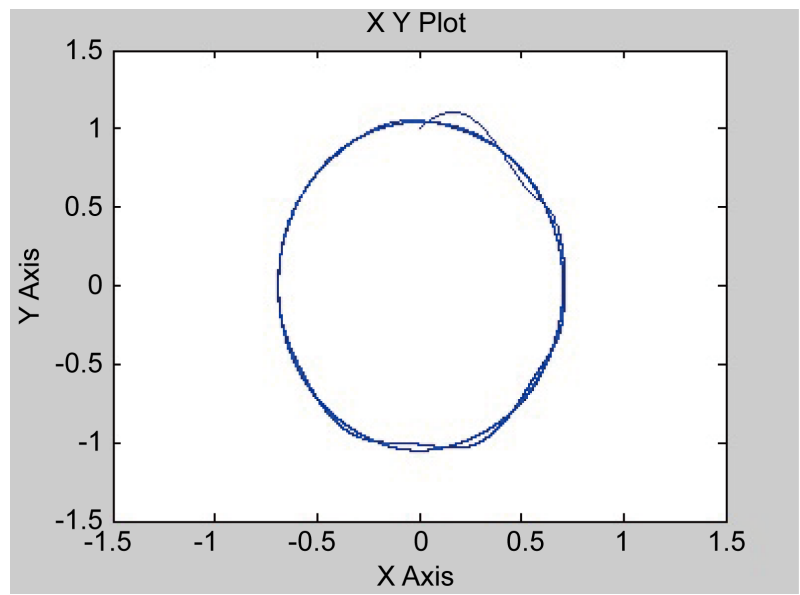

Figure 2. Duffing Actuator Position on Velocity Unstable.

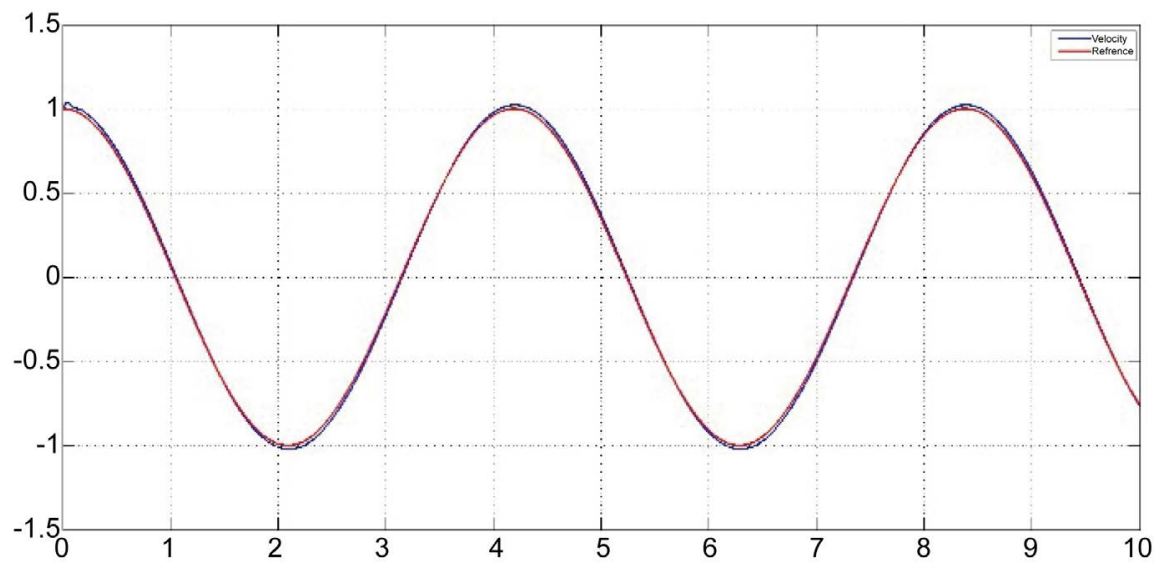

Figure 3. Duffing Stable at 25.

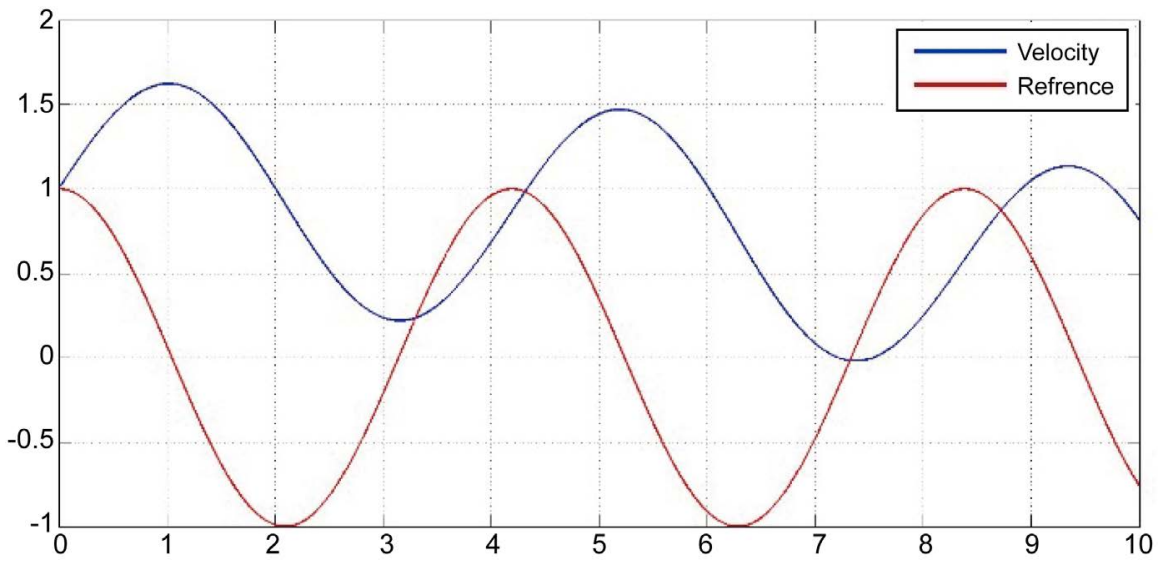

Figure 4. Duffing uncontrollable.

\subsection{Van der Pol Analysis}

The same principle and logic is applied again to the Van der Pol analysis. Equation (16) and Equation (17) represent the solution when $v$ is assumed to be $\frac{1}{2} r^{2}$ and $\frac{1}{2} r^{4}$ respectively. 


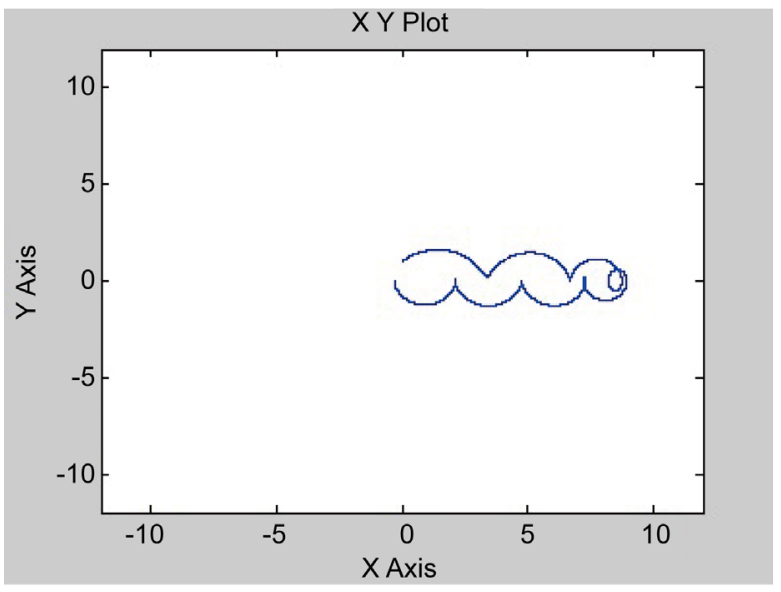

Figure 5. Duffing uncontrollable position.

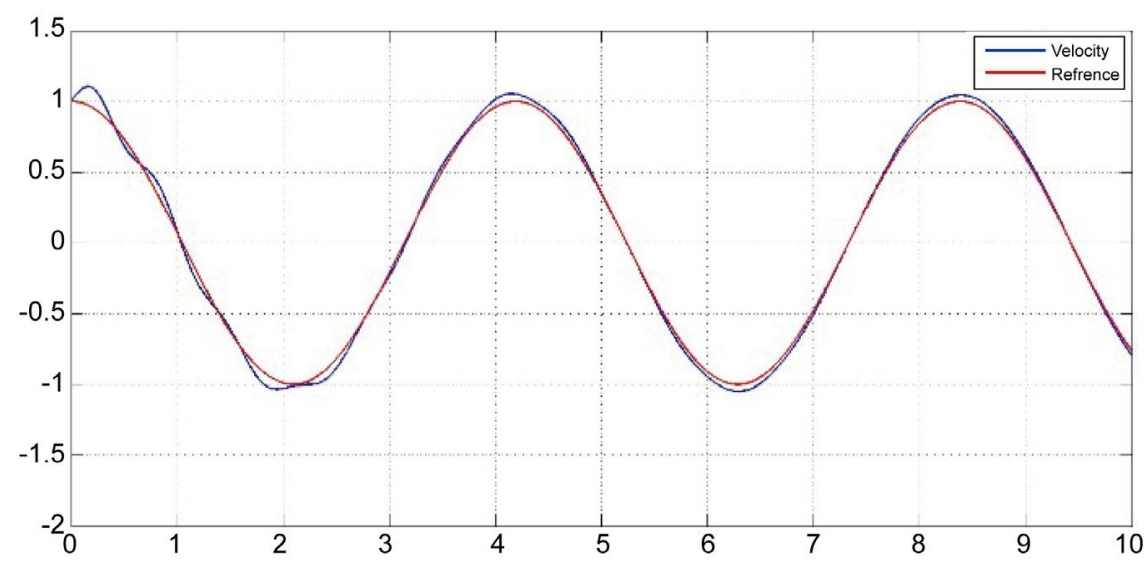

Figure 6. Duffing Unstable Velocity.

$$
\begin{aligned}
& u=\frac{k}{2} \frac{\gamma_{1}}{\gamma_{2}} e+\frac{k}{2} e^{\prime}+\frac{\gamma_{1}}{\gamma_{2}} e^{\prime}+\ddot{x}_{\text {desired }}+\alpha\left(x^{2}-1\right) x^{\prime}+x-P \cos w t \\
& u=\frac{k}{4} \frac{\gamma_{1}}{\gamma_{2}} e+\frac{k}{4} e^{\prime}+\frac{\gamma_{1}}{\gamma_{2}} e^{\prime}+\ddot{x}_{\text {desired }}+\alpha\left(x^{2}-1\right) x^{\prime}+x-P \cos w t
\end{aligned}
$$

Table 2 shows the system best performance.

Using the same flow for showing the results for the Van der Pol system. Figures 7-12 shows the same series of plots as the Duffing system. Figure 7 shows the Van der Pol Actuator Position vs. Velocity with a stable trajectory for a Van der Pol equation with sinusoidal drive. On the other hand, Figure 8 shows Van der Pol Actuator Position vs. Velocity Unstable Chaotic trajectory for a Van der Pol equation with sinusoidal drive. Again, in both cases it is assumed that $x$ is $\theta$ and $y$ is $v$. Figure 9 shows a Van der Pol Stable solution at 25 in poles with comparison to a reference sinusoidal drive. Figure 10 on the other hand, shows a Van der Pol uncontrollable system when compared to a reference sinusoidal drive at pole 7.5. Looking at the position trajectory in Figure 11, it can be noticed that it is uncontrollable position with a sinusoidal drive, it is also assumed here that that $x$ is $\theta(\theta$ : is the position $)$ and $y$ is $v$. Finally Figure 12 , shows an unstable velocity in comparison to the reference sinusoidal drive. 
Table 2. System Performance captured at these specific values.

\begin{tabular}{ccccc}
\hline $\boldsymbol{\alpha}$ & $\boldsymbol{\beta}$ & $\gamma_{1}$ & $\gamma_{2}$ & $k$ \\
\hline 0.2 & 1.2 & 3 & 0.5 & 40 \\
\hline
\end{tabular}

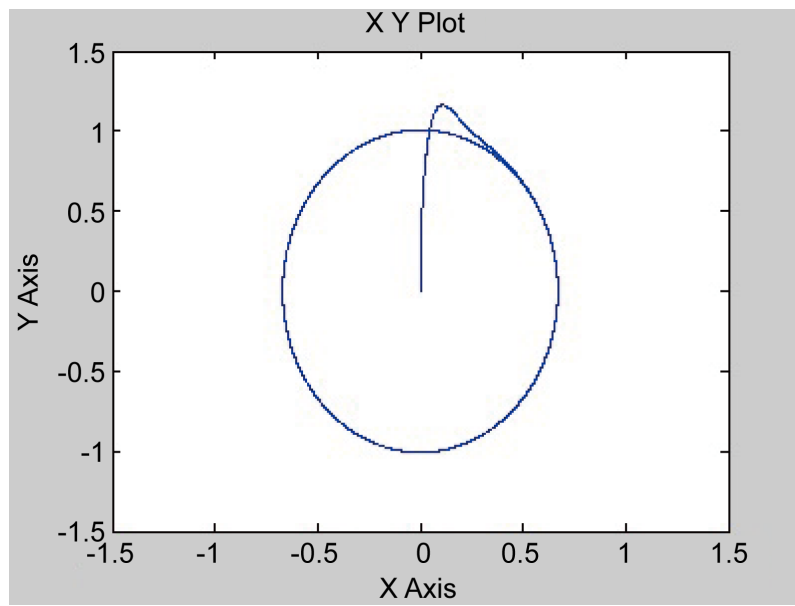

Figure 7. Van der Pol Actuator Position on Velocity Stable.

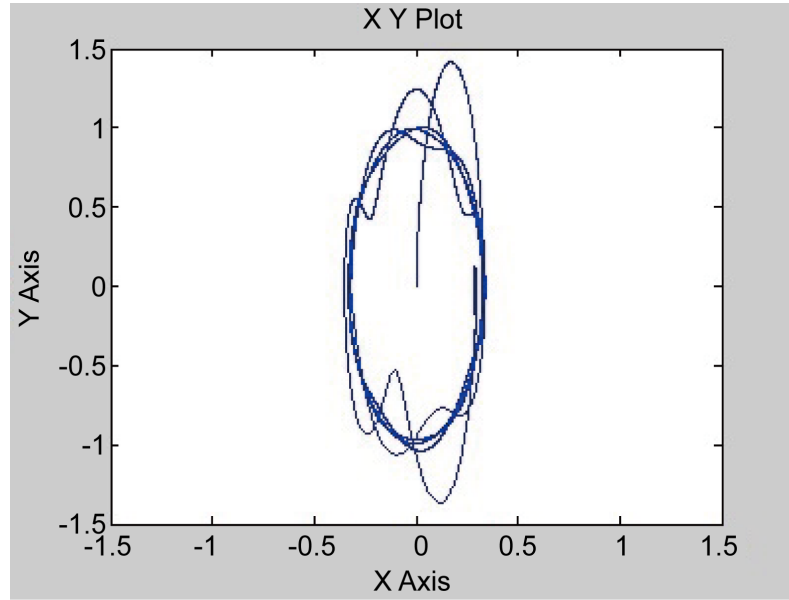

Figure 8. Van der Pol Actuator Position on Velocity Unstable.

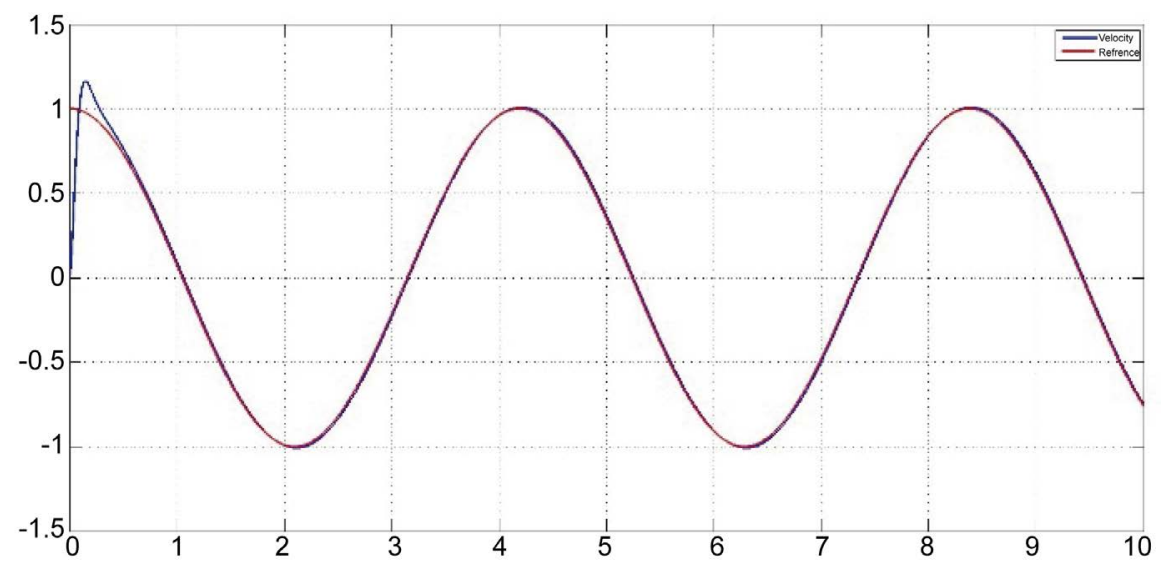

Figure 9. Van der Pol Stable at 25. 


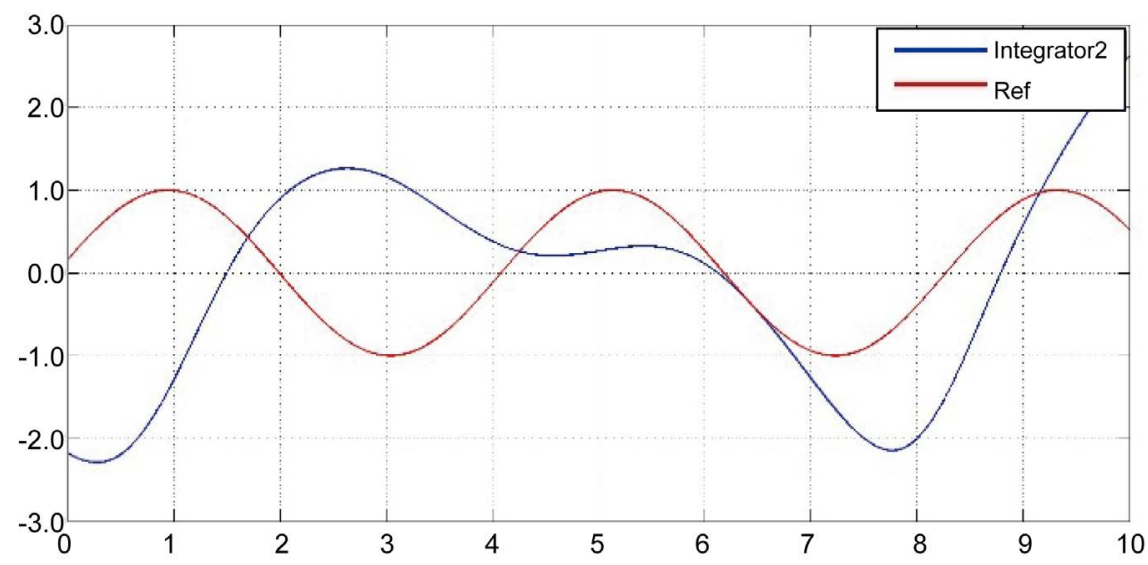

Figure 10. Van der Pol Uncontrollable.

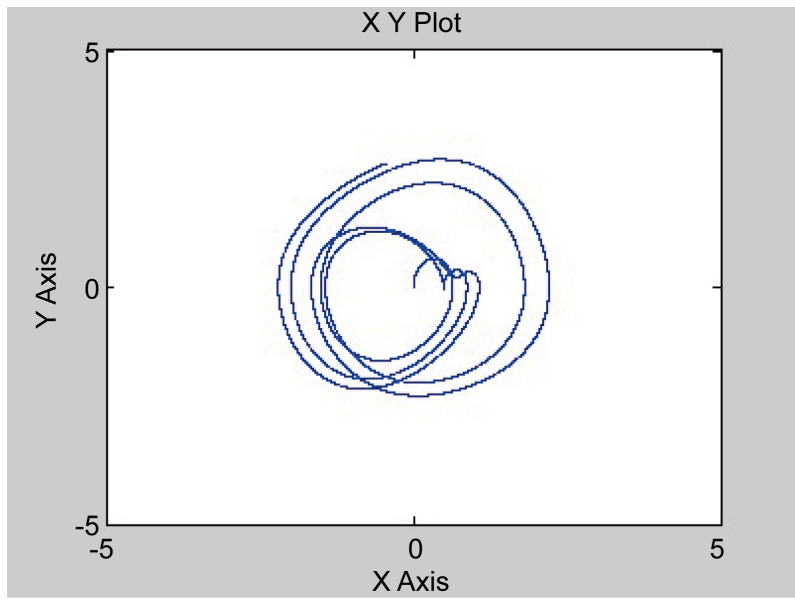

Figure 11. Van der Pol Uncontrollable Position.

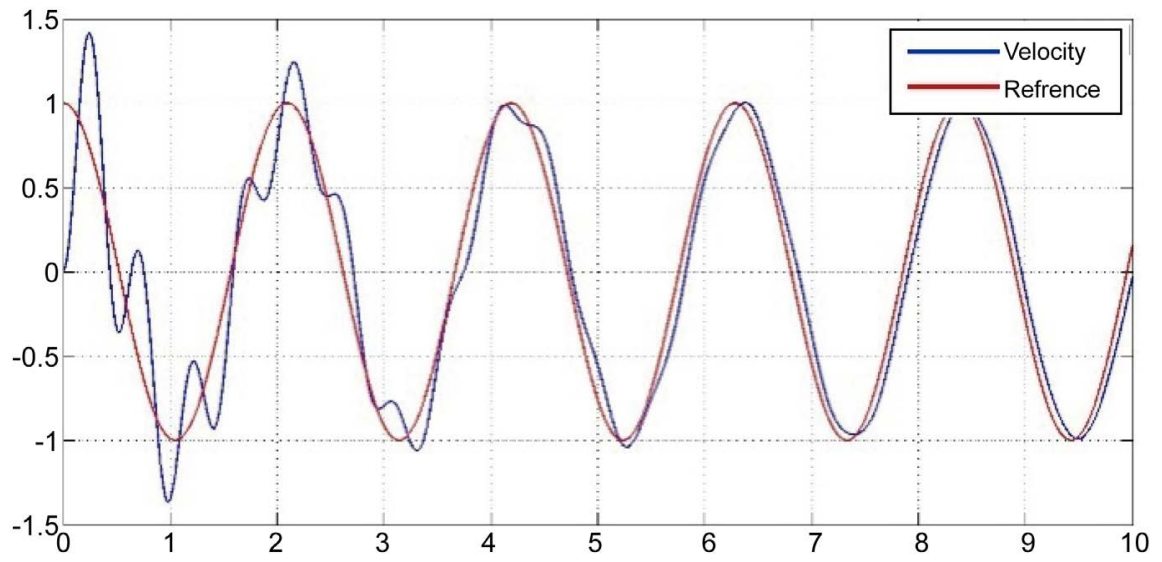

Figure 12. Van der Pol Unstable Velocity.

\subsection{Energy Calculations}

The potential and kinetic energies are studied in this section. The same analytical process is used as sections " $a$ " and " $b$ " earlier. The kinetic and the potential energy equations are shown in Equation (18) and (19) prospectively. 


$$
\begin{gathered}
K . E .=\frac{1}{2} m V^{2} \\
P . E .=\frac{1}{2} k x^{2}
\end{gathered}
$$

Using the same analysis as before the kinetic energy solution for both Duffing and Van der Pol oscillator, respectively, can be expressed as shown in Equations (20) and (21).

$$
\begin{aligned}
& u=\frac{1}{\ddot{x}}\left[\ddot{z}_{d}+\delta \ddot{x} \ddot{x}+\alpha x \ddot{x}+\beta x^{3} \ddot{x}-\dot{x} \dot{u}+\delta \dot{x}^{2} \ddot{x}+\alpha \dot{x}^{2}-3 \beta x^{2} \dot{x}^{2}+\alpha_{2} \dot{e}+\frac{k}{2}(\dot{e}+\alpha e)\right](20) \\
& u=\frac{1}{\ddot{x}}\left[\ddot{z}_{d}+\frac{k}{2}(\dot{e}+\alpha e)+\alpha\left(x^{2}-1\right) \ddot{x} \dot{x}+\ddot{x} x+\dot{x} \dot{u}-\alpha \dot{x}\left(x^{2}-1\right) \ddot{x}-\dot{x}^{2}-2 \alpha \dot{x}^{2} x^{2}+\alpha \dot{e}\right]
\end{aligned}
$$

Using similar analogy, the potential energy for a Duffing and Van der Pol oscillator, respectively, can be expressed as shown in Equations (22) and (23).

$$
\begin{aligned}
& u=\frac{1}{k \dot{x}}\left[\ddot{z}_{d}+k \alpha\left(x^{2}-1\right) \dot{x}^{2}+k x \dot{x}+k \dot{x}+\alpha \dot{e}+\frac{k}{2}(\dot{e}+\alpha e)\right] \\
& u=\frac{1}{k \dot{x}}\left[\ddot{z}_{d}+k \delta \dot{x}^{2}+\alpha k x \dot{x}+k \beta x^{3} \ddot{x}+k \dot{x}+\alpha \dot{e}+\frac{k}{2}(\dot{e}+\alpha e)\right]
\end{aligned}
$$

The dissipation energy for both the Duffing and the Van der Pol oscillators can be expressed as shown in Equation (24).

$$
\dot{D}=c \dot{x}^{2}
$$

This will yield dissipation energy solution as shown in Equations (25) and (26) representing the Van der Pol and Duffing respectively.

$$
\begin{aligned}
& u=\frac{1}{2 c \dot{x}}\left[\ddot{z}_{d}+2 c \alpha\left(x^{2}-1\right) \dot{x}^{2}+2 c x \dot{x}+\alpha \dot{e}+\frac{k}{2}(\dot{e}+\alpha e)\right] \\
& u=\frac{1}{2 c \dot{x}}\left[\ddot{z}_{d}+2 c \delta \dot{x}^{2}+2 c \alpha x \dot{x}+2 c \beta x \dot{x}^{3}+\alpha \dot{e}+\frac{k}{2}(\dot{e}+\alpha e)\right]
\end{aligned}
$$

The system best performance was then recorded at the values below:

Table 3 shows the Duffing forced;

Table 4 shows the Van der Pol forced;

Table 5 shows the Duffing unforced values;

Table 6 shows the Van der Pol unforced values.

Using these solutions the results are shown in Figures 13-15. Figure 13 shows

Table 3. System Performance captured at these specific values.

\begin{tabular}{ccccc}
\hline $\boldsymbol{\alpha}$ & $\boldsymbol{\Sigma}$ & $\boldsymbol{\beta} 1$ & $\boldsymbol{\beta} 2$ & $\gamma$ \\
\hline 1 & 0.1 & 1.5 & 1 & 1 \\
\hline
\end{tabular}

Table 4. System Performance captured at these specific values.

\begin{tabular}{ccccc}
\hline$\sigma 1$ & $\sigma 2$ & $\gamma 1$ & $\gamma 2$ & $\beta$ \\
\hline 1 & -0.1 & 1 & 1 & 0 \\
\hline
\end{tabular}


Table 5. System Performance captured at these specific values.

\begin{tabular}{ccccc}
\hline$\sigma 1$ & $\alpha$ & $\beta 1$ & $\beta 2$ & $\gamma$ \\
\hline 0.2 & 1 & 1 & 0.5 & 0 \\
\hline
\end{tabular}

Table 6. System Performance captured at these specific values.

\begin{tabular}{ccccc}
\hline$\alpha$ & $\sigma 2$ & $\sigma 2$ & $\gamma$ & $\beta$ \\
\hline 1 & 0.1 & -0.1 & 1 & 0 \\
\hline
\end{tabular}

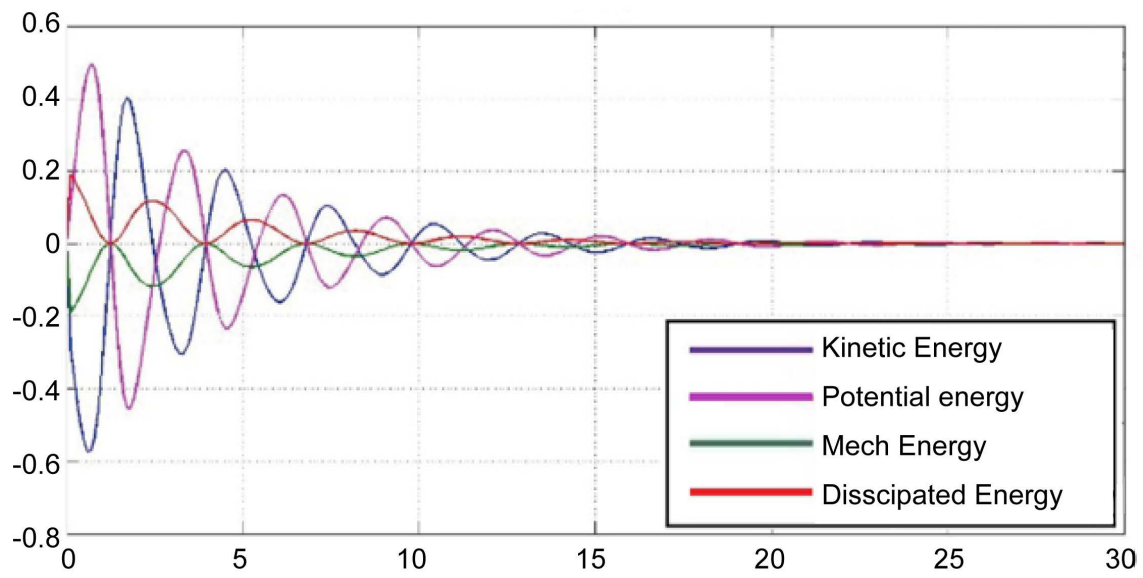

Figure 13. Duffing Energy change rate.

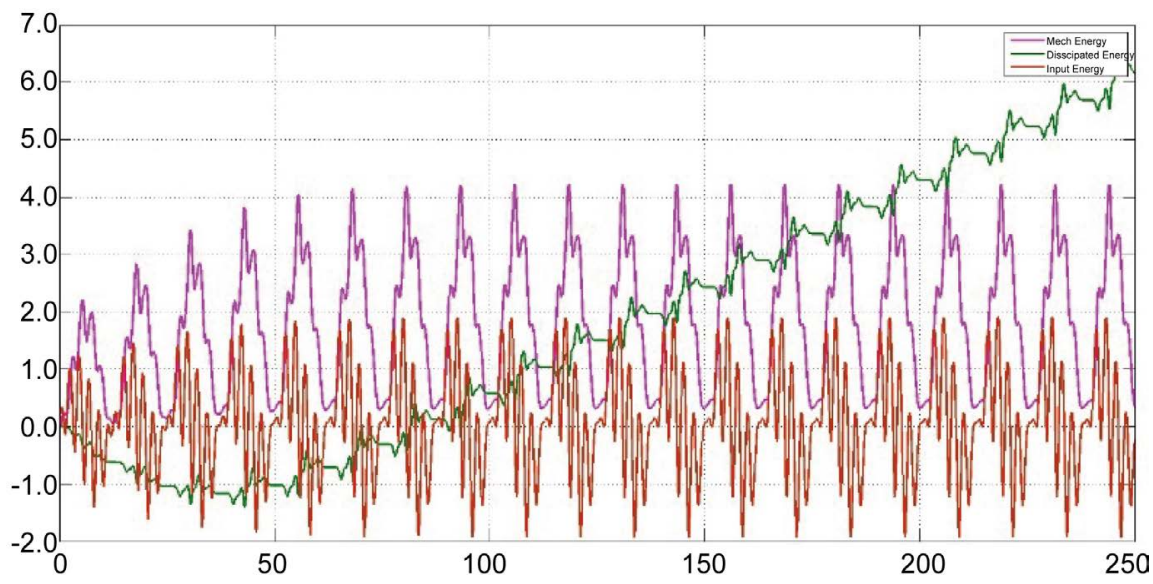

Figure 14. Forced Van der Pol.

the energy exchange rate in the unforced Duffing oscillator. This figure shows the kinetic, mechanical, and the potential energies as well as the dissipated power. Figure 14 on the other hand, shows the energy exchange in a forced Van der Pol oscillator. Again this figure shows the mechanical, input energy, and the dissipated energy. Finally, Figure 15 shows the energy exchange in an unforced Van der Pol oscillator. Figure 15 shows the kinetic, mechanical, and the potential energies as well as the dissipated power. 


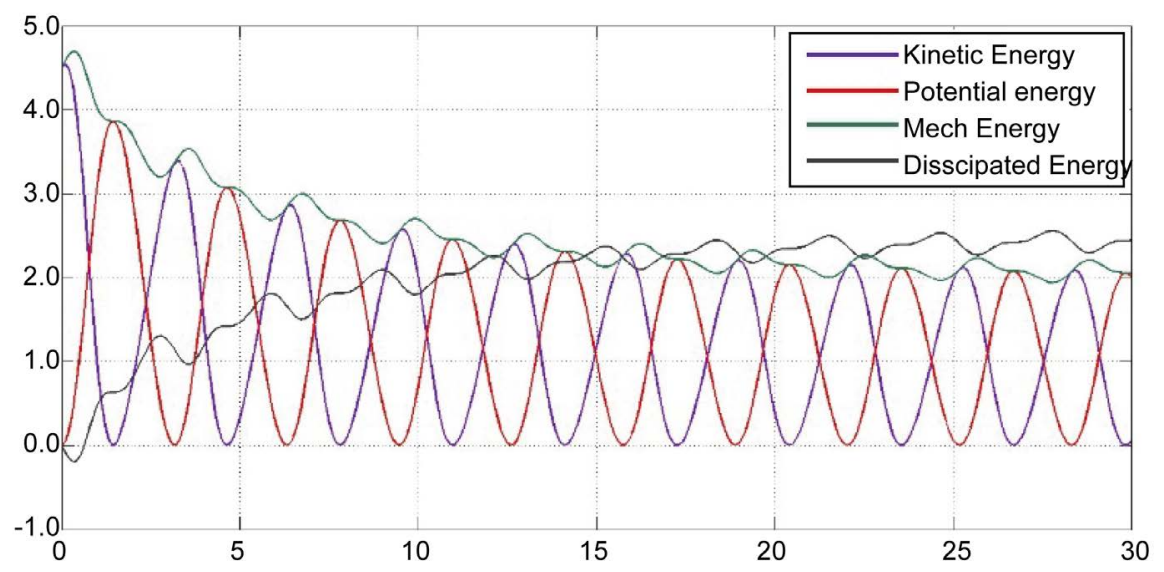

Figure 15. Unforced Van der Pol Energy.

\section{Conclusion}

In this paper, we presented a novel nonlinear control method that was applied to forced Duffing and Van der Pol oscillators that were experiencing chaotic behavior to a prescribed performance. The oscillators had an actuator applied to them. We also presented the energy exchange in forced Duffing and Van der Pol oscillators. The paper illustrated the usefulness of the presented method in the unstable areas. The presented controllers achieved two objectives: we first stabilized both the Duffing oscillator and the Van der Pol oscillators. Secondly, we presented the transient performance of the system. Robustness can be added to the system as a future work. This can be achieved by incorporating states estimator, or parameters estimator or even both. These added estimators can be integrated into the design by introducing more virtual control constraints and changing the corresponding Lyapunov function. As an additional future work, we would like to incorporate the effort of this work into another work that we did earlier and more specifically to the photovoltaic system control with the presence of an electric vehicle and a home load as we showed in [17] [18] and [19].

\section{References}

[1] Thompson, J.M.T. and Stewart, H.B. (2002) Nonlinear Dynamics and Chaos. John Wiley \& Sons, New York.

[2] Zaher, A.A., Harb, A.M. and Zohdy, M.A. (2004) Recursive Backstepping Control of Chaotic Duffing Oscillators. Proceedings of the 2004 American Control Conference, 5, 4302-4306.

[3] Harb, A., Zaher, A. and Zohdy, M. (2002) Nonlinear Recursive Chaos Control. Proceedings of the 2002 American Control Conference, 8-10 May 2002. https://doi.org/10.1109/acc.2002.1023974

[4] Bermúdez-Gómez, C.R., Enriquez-Caldera, R. and Martínez-Carballido, J. (2012) Chirp Signal Detection Using the Duffing Oscillator. CONIELECOMP 2012, 22 nd International Conference on Electrical Communications and Computers, Cholula, 344-349. https://doi.org/10.1109/conielecomp.2012.6189936

[5] Zaher, A.A. (2011) Secure Communication Using Duffing Oscillators. IEEE Inter- 
national Conference on Signal and Image Processing Applications (ICSIPA), Kuala Lumpur, 563-568. https://doi.org/10.1109/ICSIPA.2011.6144150

[6] Li, J. and Shen, Y. (2009) The Study of Weak Signal Detection Using Duffing Oscillators Array. IEEE Circuits and Systems International Conference on Testing and Diagnosis, Chengdu, 28-29 April 2009, 1-4.

https://doi.org/10.1109/cas-ictd.2009.4960901

[7] Wang, G.Y. and He, S.L. (2003) A Quantitative Study on Detection and Estimation of Weak Signals by Using Chaotic Duffing Oscillators. IEEE Transactions on Circuits and Systems I: Fundamental Theory and Applications, 50, 945-953. https://doi.org/10.1109/TCSI.2003.812606

[8] Wang, Y., Li, H. and Dai, W. (2016) Application of Duffing Oscillator in Ship Propeller Blade Number Recognition. IEEE/OES China Ocean Acoustics (COA), Harbin, 1-5.

[9] Peluffo-Ordóñez, D.H., Rodríguez-Sótelo, J.L., Revelo-Fuelagán, E.J., OspinaAguirre, C. and Olivard-Tost, G. (2015) Generalized Bonhoeffer-Van der Pol Oscillator for Modelling Cardiac Pulse: Preliminary Results. IEEE 2nd Colombian Conference on Automatic Control (CCAC), Manizales, 1-6.

[10] Menzel, K.O., Bockwoldt, T., Arp, O. and Piel, A. (2013) Modeling Dust-Density Wave Fields as a System of Coupled van der Pol Oscillators. IEEE Transactions on Plasma Science, 41, 735-739. https://doi.org/10.1109/TPS.2012.2227497

[11] Kuo, C.L., Pai, N.S., Liang, S.M. and Hu, S.H. (2008) Fuzzy Sliding-Model Control for Synchronization of an Uncertain Duffing-Holmes System. 2008 IEEE International Symposium on Knowledge Acquisition and Modeling Workshop, Wuhan, 21-22 December 2008, 104-107. https://doi.org/10.1109/KAMW.2008.4810435

[12] Jimenez-Triana, A., Tang, W.K.-S., Chen, G. and Gauthier, A. (2010) Chaos Control in Duffing System Using Impulsive Parametric Perturbations. IEEE Transactions on Circuits and Systems II: Express Briefs, 57, 305-309. https://doi.org/10.1109/TCSII.2010.2043464

[13] Guckenheimer, J. and Holmes, P. (1983) Nonlinear Oscillations, Dynamical Systems, and Bifurcations of Vector Fields. Springer-Verlag, New York. https://doi.org/10.1007/978-1-4612-1140-2

[14] Chuan-Bo, R., Zhen, Z. and Lin, L. (2011) Bifurcation and Chaos Control of Van Der Pol System with Delay. 2011 Chinese Control and Decision Conference (CCDC), Mianyang, 23-25 May 2011, 957-963. https://doi.org/10.1109/ccdc.2011.5968322

[15] Yang, Z., Jiang, T. and Jing, Z. (2010,) Chaos Control in Duffing-Van Der Pol System. 2010 International Workshop on Chaos-Fractal Theories and Applications, Kunming, 29-31 October 2010, 106-110. https://doi.org/10.1109/IWCFTA.2010.32

[16] Xiao, M., Zheng, W.X., Wan, Y., Fan, C. and Jiang, G. (2014) Bifurcation Control of an Incommensurate Fractional-Order Van Der Pol Oscillator. Proceedings of the 33 rd Chinese Control Conference, Nanjing, 28-30 July 2014, 2206-2211.

[17] Alghassab, M. and Zohdy, M. (2016) Modelling of a Solar Energy Optimized System for a Residential Setup. International Journal of Advanced Research in Science and Technology, 5, 536-543.

[18] Alghassab, M. and Zohdy, M. (2016) Modelling of a Residential Solar Energy Recuperation System Setup. Open Journal of Energy Efficiency, 5, 135-147.

[19] Alghassab, M. and Zohdy, M. (2016) Geographic Based Analysis of a Photovoltaic System in a Residential Setup. Proceedings of 2016 Universal Technology Management Conference (UTMC), Minnesota, 26-28 May 2016, 56-61. 
Submit or recommend next manuscript to SCIRP and we will provide best service for you:

Accepting pre-submission inquiries through Email, Facebook, LinkedIn, Twitter, etc. A wide selection of journals (inclusive of 9 subjects, more than 200 journals)

Providing 24-hour high-quality service

User-friendly online submission system

Fair and swift peer-review system

Efficient typesetting and proofreading procedure

Display of the result of downloads and visits, as well as the number of cited articles Maximum dissemination of your research work

Submit your manuscript at: http://papersubmission.scirp.org/

Or contactijmnta@scirp.org 